\title{
Predictive Risk Factors for Intra- and Postoperative Complications in 526 Laparoscopic Sigmoid Resections due to Recurrent Diverticulitis: A Multivariate Analysis
}

\author{
Philipp Kirchhoff • Daniel Matz • Selim Dincler • \\ Peter Buchmann
}

Published online: 24 December 2010

(C) Société Internationale de Chirurgie 2010

\begin{abstract}
Background Laparoscopic sigmoid resection is a feasible and frequent operation for patients who suffer from recurrent diverticulitis. There is still an ongoing debate about the optimal timing for surgery in patients who suffer from recurrent diverticulitis episodes. In elective situations the complication rate for this procedure is moderate, but there are patients at high risk for perioperative complications. The few identified risk factors so far refer to open surgery. Data for the elective laparoscopic approach is rare. The objective of this study was to identify potential predictive risk factors for intra- and postoperative complications in patients who underwent laparoscopic sigmoid resection due to diverticular disease.

Methods Uni- and multivariate analyses of a prospectively gathered database (1993-2006) were performed on a consecutive series of 526 patients who underwent laparoscopic sigmoid resection due to recurrent diverticulitis in a single institution. Patients were assessed for demographic data, operative indications, and intra- and postoperative complications. Altogether, we analyzed 17 potential risk factors to identify significant influence on the intra- and postoperative outcome, including timing of surgery.

Results Statistical analysis of specific medical and surgical complications revealed anemia, previous myocardial

Philipp Kirchhoff and Daniel Matz contribute equally to the work.

P. Kirchhoff · S. Dincler · P. Buchmann

Department of Surgery, City Hospital Waid, Tièche-Strasse 99,

8037 Zurich, Switzerland

P. Kirchhoff $(\bowtie) \cdot$ D. Matz

Department of Surgery, University of Basel, Spitalstrasse 21,

4031 Basel, Switzerland

e-mail: pkirchhoff@hotmail.com
\end{abstract}

infarction, heart failure, experience of the surgeon, and male gender, as independent predictive risk factors for postoperative complications. Patients older than age 75 years was the only independent risk factor for intraoperative complications in a multiple logistic regression model. Early elective surgery led to increased conversion rate but did not influence the postoperative complication rate.

Conclusions This large, single-center study provides first evidence of the significance of specific predictive risk factors for intra- and postoperative complications in laparoscopic sigmoid resection for diverticular disease.

\section{Introduction}

Today laparoscopic colorectal procedures are well established. Advantages, such as decreased length of hospital stay, reduced need for analgetics, faster onset of postoperative activity, less wound infections and lower incisional hernia rate, are well documented [1,2]. The decision for a minimal invasive approach remains challenging, and the risk of complications must be taken into account against the advantages of reduced invasiveness. Although it is well proven that laparoscopic surgery can be performed efficiently for a broad spectrum of diseases, it is still necessary to evaluate the best surgical treatment in every individual case. Many factors are used to determine whether a patient will tolerate a particular surgical procedure. There is still a lack of objective means of preoperative assessment for individual patients to choose the best surgical procedure. Perioperative complications occur with a relative risk dependent on multiple factors. Many of these factors have recently been investigated and some of them are widely accepted as risk factor for intra- or postoperative 
complications. In our recent study, we confirmed six predictive risk factors of laparoscopic colorectal surgery. This study included a variety of laparoscopic procedures for colorectal diseases [3]. Therefore, objective means to determine the risk for the individual patient undergoing a specific surgical procedure are strongly required.

Laparoscopic sigmoid resection is commonly accepted for the treatment of patients who suffer from recurrent diverticulitis. The optimal timing for surgical intervention is still discussed controversially $[4,5]$. The early elective laparoscopic sigmoid resection in the same hospital stay seems feasible; however, well-designed studies with strong evidence proving this thesis are missed in the literature. In complicated diverticulitis and emergency situations, the morbidity and mortality rate is quite high, although a few recently published studies describe the feasibility of the laparoscopic approach even in these cases [6]. In elective laparoscopic sigmoid resection, the complication rate is moderate. Therefore, we analyzed 17 potential risk factors, including the timing of surgery from a prospective data collection (1993-2006) of 526 patients who underwent elective laparoscopic sigmoid resection, to identify significant influence on perioperative medical and surgical complications.

\section{Materials and methods}

A consecutive series of patients who underwent laparoscopic sigmoid resection from 1993 through 2006 was prospectively entered into a database in the Department of Surgery at the City Hospital Waid, Zurich, Switzerland. City Hospital Waid is a teaching hospital that provides training in general surgery and laparoscopic techniques. The age of the study population ranged from 31 to 93 (mean, 64.2) years. All 526 operations were performed by 13 different surgeons; 2 of them operated on 337 cases. Surgeons with more than 70 performed laparoscopic colorectal procedures were defined as experienced [7]. They all used the same technique: trocar placement (diamond position) and circular stapled end-to-end anastomosis. Patients received a single shot of a third-generation cephalosporin preoperatively. Low-anterior resections and left hemicolectomy due to extended diverticular disease were excluded.

Indication for surgery was a history of at least two episodes of diverticulitis diagnosed by a CT scan and confirmation of diverticular disease by an endoscopy of the colon. The number of individuals with more than two episodes was not separately recorded. A total 165 patients underwent early elective surgery within 8 days after admission when suffering from ongoing pain or inflammation despite antibiotic treatment. Elective surgery after
4-6 weeks time was performed in 361 patients, who immediately responded to antibiotic treatment.

On admission, all patients received antibiotic therapy intravenously. Only cases with intraoperative classified Hinchey I score during the early elective situation were enrolled in the study. Patients classified as Hinchey II were excluded because their sample size $(n=24)$ was too small for proper analysis. Patients with Hinchey III and IV underwent open emergency surgery and were excluded. Because the study was designed to assess risk factors for laparoscopic resection of sigmoid bowel as primarily approach in our institution, the number of open resections was not recorded.

Data recorded for each patient included age, sex, body mass index, surgeon's experience, conversion to laparotomy, length of operation, intraoperative and postoperative complications, American Society of Anaesthesiology (ASA) score, preoperative haemoglobin, length of hospital stay, mortality, reoperation and multiple comorbidities according to the Charleston Index, such as diabetes mellitus, history of heart failure, or myocardial infarction, chronic obstructive pulmonary disease, cirrhosis, renal insufficiency, dementia, AIDS, leukemia, and vascular disease. The demographic and clinical data are given in Table 1.

We recorded intraoperative complications, such as bowel and ureter injury, bleeding, anastomotic problems, and anesthesiologic complications. Anastomotic problems, bowel, and ureter injury were defined as problems when further surgical procedures were needed in the same operation. Anastomotic problems were defined as failure of the stapling procedure, incomplete stapling rings, and tension of the anastomosis with further surgical intervention. Bleeding as an intraoperative complication was recorded when additional treatment, such as blood transfusion, vascular surgery, or conversion to open surgery, was applied. For anesthesiologic complications, any additional treatment by the anesthesiologist or conversion due to anesthesiological complications were documented.

Table 1 Demographics and clinical characteristics of patients

\begin{tabular}{ll}
\hline Age (years) & $64.2 \pm 11.78$ \\
Men/woman $(n)$ & $198 / 328$ \\
BMI & $26.1 \pm 4.52$ \\
Obesity BMI $\geq 30$ & $93(17.7 \%)$ \\
Malnutrition BMI $\leq 17$ & $7(1.3 \%)$ \\
Operating time (min) & $195.231 \pm 64.23$ \\
Hemoglobin (g/l) & $14.4 \pm 6.04$ \\
Length of stay (days) & $8.74 \pm 5.27$ \\
Morbidity & $85(16.2 \%)$ \\
Mortality & $4(0.07 \%)$
\end{tabular}

BMI Body mass index 
Postoperative complications were divided into surgical complications, such as bleeding, abscess, anastomotic leakage, surgical site infection, or wound dehiscence, and medical complications, such as pneumonia, pulmonary embolism, thrombosis, cardiac failure or infarction, and renal failure. Postoperative bleeding was recorded if reoperation was necessary. Deep abscess was defined by detection through ultrasound or CT scan followed by reoperation, antibiotic therapy, or CT-guided drainage. Anastomotic insufficiency diagnosed by CT scan and treated with antibiotics or reoperation was included. Surgical-site infection was defined by clinical signs and open wound treatment with or without antibiotic usage. Deep wound dehiscence was recorded when surgical reintervention was necessary. Medical complications were included if diagnosed clinically and verified by objective diagnostics and/or additional medical treatment.

To identify potential predictive risk factors for intra- and postoperative complications, we used univariate and multivariate analysis. We separately analyzed medical and surgical complications.

\section{Statistics}

Statistical analysis was performed using the SPSS statistical software package (SPSS Inc., Chicago, IL). The univariate relation between each variable and intra- and postoperative complications was evaluated using a single factor logistic model for continuous variables and Pearson's chi-squared test for categorical variables. Independent variables in the univariate analysis were entered into the multivariate logistic regression model. $P$ values $<0.05$ were considered to be statistically significant.

\section{Results}

Between 1993 and 2006, 526 laparoscopic rectosigmoid resections were performed at our department. There were
198 men and 328 women, the mean body mass index (BMI) was $26.1 \pm 4.52$, and $17.5 \%(n=93)$ were overweight $(\mathrm{BMI}>30)$ and $1.3 \% \quad(n=7)$ were underweight (BMI < 17). The average operating time was $195.231 \pm$ $64.23 \mathrm{~min}$. The mean in-hospital duration of postoperative recovery was $8.74 \pm 5.27$ days. Primarily, in none of the cases, a defunctioning stoma was necessary.

The 30-day postoperative morbidity rate was $16.2 \%$ $(n=85)$. The intraoperative complication rate was $4.9 \%$ $(n=26)$. Conversions were registered in $10.6 \%(n=56)$ of the cases. The conversion group had a significant higher intra- and postoperative complication rate compared with the laparoscopic group $(16.07 \%$ vs. $5.96 \%, P<0.001$; $32.14 \%$ vs. $14.26 \%, P=0.001)$. The demographic data and conversion rate is shown in Tables 1 and 2 .

Patients operated as early elective had a significantly higher conversion rate than the elective group (17.6\% vs. $7.5 \%, P=0.001)$. The complication rate in these two groups did not differ significantly (Table 3). Intraoperative Hinchey II classified patients $(n=24)$ had increased intraoperative (16.75\%, 2 bowel lesions, 1 ureter injury, 1 anastomotic problem) and postoperative (33.3\%, surgical complications: 3 wound complications, 1 pelvic abscess; medical complications: 1 patient suffered from deep vein thrombosis with lung embolism) complication rate. The conversion rate in these patients was $54.2 \%$. With respect to the small sample size, this group was not included in the main analysis.

Twenty-eight intraoperative complications were observed in 26 patients (4.9\%): 8 bowel lesions, 5 bleeding episodes, 5 anastomotic problems, 1 ureter injury, and 5 anesthesiological complications. In two cases, more than one complication occurred and four other complications were documented. Twelve colovesical fistulas were detected during surgery. The bladder was closed by a running suture after resection of the colon.

Altogether, 89 postoperative complications occurred in 85 patients (16.2\%), including 20 complications $(3.8 \%)$ of the converted group. The most frequent surgical complications in both groups were anastomotic leakages,
Table 2 Summary of intra- and postoperative complications and rate of conversion

Table 3 Influence of timing of surgery on complication and conversion rate

\begin{tabular}{llcc}
\hline & $\begin{array}{l}\text { Laparoscopic sigmoid } \\
\text { resection }(n=470 ; 89.4 \%)\end{array}$ & $\begin{array}{l}\text { Conversion } \\
(n=56 ; 10.6 \%)\end{array}$ & $P$ value \\
\hline Intraoperative complications & $28(5.96 \%)$ & $9(16.07 \%)$ & 0.001 \\
Postoperative complications & $67(14.26 \%)$ & $18(32.14 \%)$ & 0.001 \\
\hline
\end{tabular}

\begin{tabular}{llll}
\hline & $\begin{array}{l}\text { Early elective surgery } \\
(n=165)\end{array}$ & $\begin{array}{l}\text { Elective surgery } \\
(n=361)\end{array}$ & $P$ value \\
\hline Conversion rate & $29(17.6 \%)$ & $27(7.5 \%)$ & 0.001 \\
Intraoperative complications & $13(7.9 \%)$ & $15(4.2 \%)$ & 0.094 \\
Postoperative complications & $30(18.2 \%)$ & $55(15.2 \%)$ & 0.444 \\
\hline
\end{tabular}


Table 4 Intraoperative complications

\begin{tabular}{ll}
\hline Intraoperative complications & $n=28(5.3 \%)$ \\
\hline Bowel injury & $8(28.6)$ \\
Ureter injury & $1(3.6)$ \\
Bleeding & $5(17.6)$ \\
Anastomotic problems & $5(17.6)$ \\
Anesthesia problems & $5(17.6)$ \\
More than one complication & $2(7.1)$ \\
Other & $4(14.2)$ \\
\hline
\end{tabular}

Table 5 Postoperative complications

\begin{tabular}{ll}
\hline Surgical complications & $n=55(10.5 \%)$ \\
Anastomotic leakage & $16(29.1)$ \\
Surgical site infection & $15(27.3)$ \\
Bleeding & $10(18.2)$ \\
Pelvic abscess & $5(9.1)$ \\
Wound dehiscence & $3(5.5)$ \\
More than one & $2(3.6)$ \\
Other & $6(10.9)$ \\
Medical complications & $n=30(5.7 \%)$ \\
Pneumonia & $15(50)$ \\
Cardiac complications & $6(20)$ \\
Pulmonary embolism & $2(6.7)$ \\
Deep vein thrombosis & $2(6.7)$ \\
Other & $5(16.7)$ \\
More than one complication & $5(16.7)$ \\
\hline
\end{tabular}

postoperative bleeding, and surgical-site infections. Pneumonia and cardiac complications were the major medical postoperative problems. The 30-day mortality rate was $0.07 \%(n=4)$. Detailed intra- and postoperative complications are listed in Tables 4 and 5.

Table 6 shows the perioperative potential risk factors recorded prospectively from 1993 to 2006. These were analyzed to detect their influence on intra- and postoperative complications (Tables 6, 7 and 8).

\section{Univariate analysis}

Experienced surgeons had significantly less postoperative complications $(15.8 \%$ in the experienced group vs. $27 \%$ in the less experienced group, $P=0.025$ ). Intraoperative complications did not differ significantly between these groups.

Patients older than 75 years had more intraoperative complications than younger ones $(3.5 \%$ in younger group vs. $14.8 \%$ in the group $>75$ years, $P=0.001)$.

Obese $\quad(\mathrm{BMI}>30) \quad$ or malnourished $\quad(\mathrm{BMI} \leq 17)$ patients were not at significantly higher risk for intra- and postoperative complications. Postoperative complications were observed more often in men than in women $(28.6 \%$ vs. $14.3 \%, P=0.003$ ). The patient group graded ASA III and higher were not at significant risk to develop intraoperative or postoperative problems.

Preoperative anemia $(\mathrm{HB} \leq 12 \mathrm{~g} / \mathrm{l})$ had significant influence on intraoperative complications $(P=0.01)$, although postoperative morbidity was not influenced.

Interestingly, presence of liver disease led to significant elevated postoperative problems $(P=0.02)$. Previous heart failure and previous myocardial infarction had the tendency to cause more postoperative medical problems in the univariate analysis $(P=0.069, P=0.083)$. The intraoperative course also was significantly influenced in patients with presence of dementia $(P=0.031)$. Cerebrovascular disease, renal failure, and chronic lung disease seemed to have no influence on the intra- or postoperative course in the univariate analysis (data shown in Table 6).

Multivariate analysis

Tables 7 and 8 summarize the results of the multivariate analysis. Male gender $(P=0.001)$, preoperative anemia (HB $\leq 12 \mathrm{~g} / \mathrm{l} ; \quad P=0.045)$, and previous myocardial infarction $(P=0.02)$ were independent predictive for developing postoperative surgical complications. Previous heart failure in the medical history was predictive for postoperative medical complications $(P=0.008$; Table 7). Additionally male gender $(P=0.002)$, age $>75$ years $(P=0.026)$, and experience of the surgeon $<70$ cases $(P=0.045)$ were independently predictors for overall postoperative outcome. Only age $>75$ years was an independent risk factor for intraoperative complications $(P=0.001$; Table 8$)$. These patients had significantly more anesthesiological problems compared with patients younger than 75 years $(P=0.007)$.

\section{Discussion}

The feasibility and safety of laparoscopic colorectal surgery has been reported repetitively in current surgical literature. The standard of quality and improvements in the perioperative clinical course should always be taken into consideration by the surgical team. The perioperative period can be more successful if impact factors on the patient's outcome are known. Therefore, the necessity to provide the surgeon with an objective means of assessment for individual patients especially in laparoscopic colorectal surgery is given.

One of the most performed procedures in colorectal surgery is the laparoscopic sigmoid resection due to recurrent diverticulitis. There is still a debate about the 
Table 6 Risk factors for overall intra- and postoperative complications
ASA American Society of Anaesthesiology; BMI body mass index

Bold indicates significant value

\begin{tabular}{llll}
\hline Variables & $n$ & $\begin{array}{l}\text { Intraoperative } \\
\text { complications } \\
P \text { value }\end{array}$ & $\begin{array}{l}\text { Postoperative } \\
\text { complications } \\
P \text { value }\end{array}$ \\
\hline Experience $<70 / \geq 70$ cases & & 0.26 & $\mathbf{0 . 0 2 5}$ \\
Age $\geq 75$ years & $174 / 352$ & $\mathbf{0 . 0 0 1}$ & 0.92 \\
BMI $\geq 30$ & 108 & 0.1 & 0.95 \\
BMI $\leq 17$ & 94 & 0.59 & 0.82 \\
Male/female gender & 5 & 0.83 & $\mathbf{0 . 0 0 3}$ \\
ASA score $\geq$ III & $198 / 328$ & 0.12 & 0.43 \\
Liver disease & 108 & 0.44 & $\mathbf{0 . 0 2}$ \\
Diabetes & 9 & 0.08 & 0.72 \\
Anemia HB $\leq 12$ g/l & 22 & $\mathbf{0 . 0 1}$ & 0.26 \\
Previous myocardial infarction & 56 & 0.35 & 0.069 \\
Previous heart failure & 15 & 0.57 & 0.083 \\
Vascular disease & 58 & 0.24 & 0.19 \\
Cerebrovascular disease & 23 & 0.58 & 0.52 \\
Dementia & 11 & $\mathbf{0 . 0 3 1}$ & 0.45 \\
Chronic lung disease & 3 & 0.55 & 0.1 \\
Renal failure $($ Creatinine $>260 \mu \mathrm{mol} / \mathrm{l})$ & 25 & 0.74 & 0.53 \\
\hline
\end{tabular}

Table 7 Multivariate logistic regression analysis of variable associated with medical and surgical complications

\begin{tabular}{|c|c|c|c|c|c|c|}
\hline Variable & OR & $\begin{array}{l}\text { Medical } \\
\text { complications } \\
{[95 \% \mathrm{CI}]}\end{array}$ & $P$ value & OR & $\begin{array}{l}\text { Surgical } \\
\text { complications } \\
{[95 \% \mathrm{CI}]}\end{array}$ & $P$ value \\
\hline Previous myocardial infarction & & & & 3.85 & [1.24-11.93] & 0.02 \\
\hline Anemia HB $\leq 12 \mathrm{~g} / 1$ & & & & 2.2 & {$[1.02-4.74]$} & 0.045 \\
\hline Male gender & & & & 2.44 & {$[1.41-4.21]$} & 0.001 \\
\hline Previous heart failure & 3.27 & {$[1.37-7.8]$} & 0.008 & & & \\
\hline
\end{tabular}

Table 8 Multivariate logistic regression analysis of variable associated with intra- and postoperative complications

\begin{tabular}{|c|c|c|c|c|c|c|}
\hline Variable & OR & $\begin{array}{l}\text { Intraoperative } \\
\text { complications } \\
{[95 \% \mathrm{CI}]}\end{array}$ & $P$ value & OR & $\begin{array}{l}\text { Postoperative } \\
\text { complications } \\
{[95 \% \mathrm{CI}]}\end{array}$ & $P$ value \\
\hline $\begin{array}{l}\text { Experience } \geq 70 \\
\text { cases }\end{array}$ & & & & 0.61 & [0.37-0.99] & 0.045 \\
\hline Age $\geq 75$ years & 4.27 & [1.97-9.26] & 0.001 & 1.89 & [1.08-3.32] & 0.026 \\
\hline Male gender & & & & 2.22 & [1.36-3.64] & 0.002 \\
\hline
\end{tabular}

CI confidence interval; OR odds ratio; ASA American Society of Anesthesiology

preferred approach in the face of better results comparing the early elective or the elective surgery. So far, there is no evidence that early laparoscopic sigmoid resection results in more complications than the elective procedure if strict patient selection criteria are followed, duration of the procedure is comparable to open surgery, and conversion rate is less than $10 \%$ [8]. Our study confirmed these results; although we detected a higher conversion rate in the early elective group, the complication rate did not differ.
In our series of 526 patients, using a univariate and multivariate analysis, we examined 17 perioperative and defined risk factors that proved to be significant in predicting an increase of intra- and postoperative complications. These included in the multivariate analysis the age $>75$ years, experience of the surgeon, male gender. The overall morbidity in our population was $16.2 \%$, whereas the mortality rate was $0.07 \%$. Recently reported morbidity rates between 6 and $31 \%$ for laparoscopic colorectal 
surgery are well comparable with our data. This also applies to the reported mortality rates varying between 0 and $4.5 \%$ in the published literature $[9,10]$.

No large study has been conducted to investigate the risk factors for perioperative complications undergoing laparoscopic sigmoid resection, and until now most studies have concentrated on only few factors with small sample sizes. In addition, the influence of the patient's weight on perioperative complication rates in open and laparoscopic major visceral surgery was extensively examined, but the results are still controversial $[11,12]$. In open colorectal surgery, preoperative risk factors, such as emergency operation, age $\geq 75$ years, abdominal radiation, hypoalbuminemia, COPD, previous myocardial infarction, and diabetes mellitus were validated in 1992 [13]. In our study previous myocardial infarction only increased the risk for medical complications but did not influence the rate of postoperative surgical complications. However, none of the studies differentiated between intra- and postoperative complications or medical and surgical problems. We think that this is important for the outcome and offers more precise guidance to the surgical team.

One large study with 1,421 patients, which was designed to identify risk factors for postoperative complications, combined data from open and laparoscopic surgery. They found that patient age ( $>70$ years), neurological or cardiopulmonary comorbidity, hypoalbuminemia, long duration of operation, and peritoneal contamination independently increased the risk of postoperative complications. Recently the same group published a score (AFC score) that is a pertinent predictive score of postoperative mortality after colorectal surgery. Despite the interesting results and feasibility of this score, it does not include morbidity and gives no information about intraoperative risk factors [14, 15]. Longo et al. concluded that patients with an ASA class $\geq$ III, serum sodium $>145 \mathrm{mmol} / \mathrm{l}$, and ascites had an increased morbidity or mortality rate after colectomy $[16,17]$. Because in our multivariate analysis, the predictive factors, such as age, gender, previous heart failure, and myocardial infarction, cannot be influenced perioperatively, it helps the surgeon to be aware of increased risk and therefore choose the safest treatment for the patient. The experience of the surgeon plays a significant role in complex cases. This fact favors a well-trained surgeon for a patient at risk rather than an inexperienced resident. Interestingly the univariate analysis revealed six predictive risk factors for complications. Beside correction of preoperative anemia comorbidities should be detected and properly adjusted before surgery to minimize risk. In addition the different risk factors should be kept in mind before the planed surgical procedure to avoid increasing the risk of perioperative complications.
In conclusion, this study provides first evidence of predictive risk factors for intra- and postoperative complications in patients undergoing laparoscopic sigmoid resection and differentiation of medical from surgical complications. It also elucidates the influencing risk factors on the intraoperative course in surgery. This might help the surgeon in making perioperative decisions and also could indicate that an experienced surgeon should be involved according to the preoperative risk assessment. Because patient outcome is difficult to predict, these data should guide the surgeon through the decision-making process and might lead to a feasible and simple score in the future that helps to minimize risk and maximize the patient's benefit from laparoscopic colorectal surgery. A score should draw the surgeon's attention to a high-risk patient and to an improvement of surgical outcome or change of treatment plan.

Acknowledgments Dr. Kirchhoff designed the study, analyzed the data, performed statistical analysis, and drafted the manuscript and had full access to all of the data in the study and takes responsibility for the integrity of the data and the accuracy of the data analysis. Dr. Matz performed statistical analysis and drafted the manuscript. Dr. Dincler and Prof. Buchmann participated in the design and coordination of the study. All authors read and approved the final manuscript.

\section{Conflict of interest None.}

\section{References}

1. Scala A, Huang A, Dowson HM et al (2007) Laparoscopic colorectal surgery-results from 200 patients. Colorectal Dis 9(8):701-705

2. Lezoche E, Guerrieri M, de Sanctis A et al (2006) Long-term results of laparoscopic versus open colorectal resections for cancer in 235 patients with a minimum follow-up of 5 years. Surg Endosc 20(4):546-553

3. Kirchhoff P, Dincler S, Buchmann P (2008) A multivariate analysis of potential risk factors for intra- and postoperative complications in 1316 elective laparoscopic colorectal procedures. Ann Surg 248(2):259-265

4. Zdichavsky M, Granderath FA, Blumenstock G et al (2010) Acute laparoscopic intervention for diverticular disease (AIDD): a feasible approach. Langenbecks Arch Surg 395(1):41-48

5. Zingg U, Pasternak I, Guertler L et al (2007) Early vs. delayed elective laparoscopic-assisted colectomy in sigmoid diverticulitis: timing of surgery in relation to the acute attack. Dis Colon Rectum 50(11):1911-1917

6. Titu LV, Zafar N, Phillips SM et al (2009) Emergency laparoscopic surgery for complicated diverticular disease. Colorectal Dis 11(4):401-404

7. Dincler S, Koller MT, Steurer J et al (2003) Multidimensional analysis of learning curves in laparoscopic sigmoid resection: eight-year results. Dis Colon Rectum 46(10):1371-1378

8. Tonelli F, Di CV, Liscia G et al (2009) [Diverticular disease of the colon: diagnosis and treatment. Consensus conference, 5th 
National Congress of the Italian Society of Academic Surgeons]. Ann Ital Chir 80(1):3-8

9. Degiuli M, Mineccia M, Bertone A et al (2004) Outcome of laparoscopic colorectal resection. Surg Endosc 18(3):427-432

10. Schwenk W, Haase O, Neudecker J et al (2005) Short term benefits for laparoscopic colorectal resection. Cochrane Database Syst Rev 3:CD003145

11. Gendall KA, Raniga S, Kennedy R et al (2007) The impact of obesity on outcome after major colorectal surgery. Dis Colon Rectum 50:2223-2237

12. Lascano CA, Kaidar-Person O, Szomstein S et al (2006) Challenges of laparoscopic colectomy in the obese patient: a review. Am J Surg 192(3):357-365

13. Ondrula DP, Nelson RL, Prasad ML et al (1992) Multifactorial index of preoperative risk factors in colon resections. Dis Colon Rectum 35(2):117-122
14. Alves A, Panis Y, Mantion G et al (2007) The AFC score: validation of a 4-item predicting score of postoperative mortality after colorectal resection for cancer or diverticulitis: results of a prospective multicenter study in 1049 patients. Ann Surg 246(1): 91-96

15. Alves A, Panis Y, Mathieu P et al (2005) Postoperative mortality and morbidity in French patients undergoing colorectal surgery: results of a prospective multicenter study. Arch Surg 140(3): 278-283; discussion

16. Yoo PS, Mulkeen AL, Frattini JC et al (2006) Assessing risk factors for adverse outcomes in emergent colorectal surgery. Surg Oncol 15(2):85-89

17. Longo WE, Virgo KS, Johnson FE et al (2000) Risk factors for morbidity and mortality after colectomy for colon cancer. Dis Colon Rectum 43(1):83-91 